\title{
Testing the Mental Toughness Model in the Setting of Chinese Martial Arts 中國武術運動員的心理素質
}

\author{
Veronika PARTIKOVA \\ Department of Sport and Physical Education, \\ Hong Kong Baptist University, HONG KONG
}

\begin{abstract}
BACKGROUND: Aim of this paper is to test the Sport Mental Toughness Questionnaire (SMTQ) in the setting of Chinese martial arts. The target population is adult European practitioners of modern and traditional wushu. Mental toughness is an established concept of being able to perform under pressure while remaining positive and perseverant. However, it has not yet been tested on this population. It is argued that Chinese martial arts can generate unique types of pressure situations, such as dealing with pain, body to body contact, fear of sparring, and a lack of designated levels of achievement, such as a belt ranking system.
\end{abstract}

METHODS: 277 respondents (85 female) were sampled from 28 countries. The exploratory factor analysis was conducted in SPSS, and internal consistency was calculated. Confirmatory factor analysis using AMOS was used to evaluate the model fit.

RESULTS: The EFA resulted in losing four items, with two items switching places on the components. The model reached a good fit of $\mathrm{x} 2 / \mathrm{df}=1.307$, IFI $=.965, \mathrm{CFI}=.963$ and RMSEA $=.047$.

CONCLUSIONS: This paper provided the validation of the SMTQ scale for a Chinese martial arts setting. Researching mental skills in the specific environment of Chinese martial arts may be a fruitful direction for further research, especially when developing a mental skills training program for this population. The modified model resulting from this study is the first step in this respect. Moreover, it confirms the recently discussed multidimensionality of the mental toughness model.

Key words: Mental toughness; mental skills; martial arts;

\section{Introduction}

At the starting line of any race, when comparing athletes of relatively similar levels of physical skills, mental skills may be the factor that determines the results. In a training environment, the constant pressure from rivals and need to reach a higher goal requires a significant amount of psychological strength. The ability to withstand the pressure and remain calm and positive is called mental toughness. It is defined as "unshakeable perseverance and conviction towards some goal despite pressure or adversity" (Middleton, Marsh, Martin, Richards and Perry, 2004, p. 6).
The model of mental toughness has evolved over time, with Jones's work (2002) setting the base in the sports research field. He defined mental toughness as the ability to "Generally, cope better than your opponents with the many demands (competition, training, lifestyle) that sport places on a performer. Specifically, be more consistent and better than your opponents in remaining determined, focused, confident, and in control under pressure" (p. 209), Jones developed a model of mental toughness involving 12 components. Middleton then connected mental toughness with overcoming adversity by adjusting Jones' original model (Middleton et al., 2004). In 2005 , the model was again adjusted to include self- 
presentation, which marks athlete's self-presentation to impact the opponent's self-confidence (Thelwell, Weston \& Greenlees, 2005and identify what the essential attributes required for the mentally tough soccer player are. A total of six male professional soccer players agreed to participate in the study. Semi-structured interview was conducted by the lead researcher. Having analyzed and interpreted the interview transcripts, and confirmed the findings with the participants, the following definition was generated: Mental toughness is having the natural or developed psychological edge that enables you to always cope better than your opponents with the many demands (competition, training,lifestyle). Numerous models of mental toughness have appeared since this time (Loehr, 1982; Kobasa, 1979; Clough, Earle, and Sewell, 2002; Bull, Shambrook, James and Brooks, 2005); however, a more recent one focused on a target population coming from various competition levels and sports (Sheard, Golby $\&$ van Wersch, 2009). For the purpose of this paper, this model of mental toughness was adopted.

Recently, it was also suggested that mental toughness is not a multidimensional construct, but unidimensional; these researchers pointed out that some of the components of the previous models are overlapping (Gucciardi, et al., 2015). This question of multidimensionality versus unidimensionality is currently being debated in academic circles, and this paper adds to this discussion.

The concept of pressure, as related to this discussion of mental toughness, may arise from the act of competing, fear of opponents, and the need to overcome injury, negative thoughts, and pain or exhaustion when the situation does not seem in favour of the individual. However, Chinese martial arts offer additional pressure factors: fear of fighting, being hurt or hurting someone, long-life learning without any objective measurements of improvement (such as belt ranking), pain from conditioning the bones and muscles by hitting, endless repetition of single movements, tiring stance training, full-contact sparring and partner exercises, as well as body-to-body contact.

Mental toughness was previously researched in the area of martial arts and combative sports, but the amount of research is significantly less than in other sports. Heiny (2012) highlighted well-developed mental toughness as a protective factor for aggression in judo practitioners.
Chen and Cheesman (2013) noted that direct contact fighting brings emotional and stressful demands on athletes, and in their research, they suggested that mental toughness is developed during the career of an athlete. They found a correlation between the competition level of MMA (mixed martial arts) practitioners and their level of mental toughness, which was also supported by other research (Bhardwaj, Singh, \& Rathee, 2014). Singh and Solanki (2015) tested the difference in mental toughness between judo and tae-kwon-do practitioners, and found no significant difference cause by the different style of martial art.

Whilst a sizable portion of academic research focuses on Japanese martial arts, thus far Chinese martial arts go relatively unnoticed. This can be due to the fact that the structuring of Chinese martial arts is more complicated, containing several dozen very different styles. However, Kuan and Roy (2007) conducted the first mental toughness research in this. This research found that those, who won medals at the competition, obtained scored higher in self-confidence and negative energy control. The respondents of this study were modern wushu athletes.

\section{Chinese Martial Arts}

Due to the unique environment of Chinese martial arts, there has been scholarly discussion about whether models of mental toughness from other sports could fit this population. Minnix14 framed a new model targeting Chinese traditional martial arts and, in addition to the previously established components, he added three new ones specific to Chinese traditional martial arts: anticipation, learning attitude and ethics, as additional contributing factors. With the unique demands placed on practitioners and athletes in Chinese martial arts, it is clear that mental toughness has to have a specific model for this population, which can then result in more practical and useful research. Martial arts practitioners would benefit from mental skills training based on the specific challenges they face and incorporated into their preparations. For that reason, it is necessary to explore mental toughness specifically for martial arts practitioners. This paper aims to benefit both the scholarly research community and the practitioners and athletes of Chinese martial arts. 
Chinese martial arts are divided into wushu or kung fu; however, these two branches are often confused. The International Wushu Federation recognizes two types of Chinese martial arts, or two types of wushu: modern and traditional (IWUF, n. d.).. Modern wushu is a competition-based sport with two separate divisions: taolu (forms) and sanda (fighting), with practitioners typically specializing from the beginning of their training. Taolu wushu involves artistic movements and difficult jumps, and is more similar to gymnastics or acrobatics. Sanda is a full-contact discipline, similar to kick-boxing, with allowed throws to the ground.

On the other hand, self-defence based traditional wushu, often called kung fu (Wang, 2012), has been described by Lorge (2012, p. 6) as, "At root, martial art is about skill with violence". Kung fu involves movements based on real-world application - the ability to use them in a fight. However, since its use as selfdefence has decreased since its creation, kung fu (traditional wushu) is now more well-known and sought after for its sophisticated philosophy and self-development through lifelong learning

\section{Objectives of the study}

To explore mental toughness specifically for this target group - adult European practitioners of Chinese martial artists - a recently developed mental toughness model was tested in the context of Chinese wushu, including both traditional and modern wushu practitioners. The factorial validity was tested on the three components model (control, confidence and constancy) of the Sport Mental Toughness Questionnaire, with the additional aim to examine the theory of multidimensionality of mental toughness.

\section{Materials and methods}

\section{Subjects}

This research aimed to recruit practitioners of both modern and traditional wushu, two branches of Chinese martial arts. In modern wushu, competition is one of the main goals of training and many of these practitioners are also athletes (those, who actively compete at championships). On the other hand, traditional wushu does not necessary involve competing. For that reason, practitioners of both types of wushu were invited to participate in the study, rather than only athletes.
This study targeted European adult male and female practitioners. Therefore, the first limitation of the recruitment was European citizenship. The age limit, 1845 , was set to keep representative groups of both wushu types homogenous; in modern wushu it is common for the practitioners and athletes to stop training after a certain age, similar to many other competition-oriented sports; however, age plays no such role in traditional wushu. Thus, the age limit was set to control for these differences between the two styles. Another limitation was training experience and frequency: respondents must have been training for a minimum of one month. In the end, the mean of the training experience (duration in years) was 9 years, with 35 years as the maximum value. For frequency, the typical training week had to consist of at least two sessions of at least 60-minute training.

As previously mentioned, wushu is a very broad category, and therefore it was defined with the following limitations: The practitioner had to take part in taolu training, which marks forms, or sets of movements. In addition, he or she may also train in sanda, or any form of contact sparring. While practitioners usually specialise in either taolu or sanda in modern wushu, traditional wushu has no such divisions between forms and sparring. This limitation was designed to exclude those with only fighting experience (such as with kickboxing), but no experience in other aspects of wushu. All Chinese martial arts schools of wushu were accepted: nanquan, changquan and taijiquan for modern wushu practitioners, and shaolin, wudang, emei, nanquan styles, taijiquan, xingyiquan and baguazhang for traditional wushu practitioners. Each of the specific school has its own forms (taolu) and in the case of traditional wushu, use different strategies in the sparring.

The subjects were recruited through two methods: face-to-face during fieldwork in Europe, and online. Respondents who filled in the questionnaire online were found through the following sources: social media connected with wushu; email communication with wushu schools or clubs; and visitors to the research project's website. Respondents who were recruited face-to-face were typically members of local clubs in Czech Republic or England, or athletes participating in a competition in the Slovak Republic and at the European Traditional Wushu (Kung Fu) Championship in Georgia, organized by the European Wushu Federation. The recruitment process took place during February and June 2017. 
Recruiting a representative sample of respondents proved problematic due to the unexpectedly small number of adult practitioners of modern wushu.

The final sample was $n=277$, of which 111 participated in modern wushu and 166 participated in traditional wushu. There were 85 women and 192 men. 108 respondents have competition experience at the international level. This sample was split into two groups, with the first sample used to test the EFA ( $(\mathrm{n}=139)$ and the second one to test the CFA $(\mathrm{n}=138)$. The first split group consisted of 67 modern wushu practitioners and 72 traditional wushu practitioners, and the second split consisted of 44 modern and 94 traditional.

\section{Instrument}

The Sport Mental Toughness Questionnaire (SMTQ) was used in this study. It was developed by by Sheard, Golby and van Wersch (2009), and is one of the more recent measurement tools. This questionnaire consists of 14 items based on three primary concepts: constancy, confidence and control. The items in the questionnaire are phrased as statements, such as "I interpret potential threats as positive opportunities." Answers are chosen on four-point Likert scale, ranging from "not at all true" to "very true." The concepts are measured with four items each, except for the confidence concept, which is measured with six items. The internal consistency was tested by the authors, and the Cronbach's alpha reached $.71, .74$, and .81 for the concepts. The questionnaire was tested in 25 unique sports settings with athletes of various competition levels. The author of this paper obtained permission to use questionnaire responses from all respondents.

\section{Procedure}

The questionnaire was administrated in person or online, and only in English. The original questionnaire was in English as well, and therefore no translation was needed. Each participant received detailed information about the ethical approval of the study from the author's affiliated university, their option of leaving the project at any time, and how their data will be handled.

Several items from the scale were originally negatively scored. After data collection and data input, the coding for these items was reversed. These are items 9-14, specifically. The data was screened for missing values prior to any analysis. There was a high level of respondents, despite not including any incentives for completing the questionnaire. The test of data showed less than a 5\% skip rate, which would not have any significant influence on the results (Schafer, 1999; Bennett 2001). The missing Likert scale values were replaced with the median.

\section{Statistical Analysis}

The study was divided into two parts. In Study 1, the exploratory factor analysis (EFA) was run to test the concepts and their item loadings, and served to confirm or reject the original model. In Study 2, the confirmatory analysis (CFA) was carried out to test the model fit. Cronbach's alpha was used to test the scale's reliability. The EFA analysis was performed using SPSS software, whereas the CFA was performed using AMOS software.

\section{Results}

\section{Exploratory Factor Analysis}

The first sample $(n=139)$ was used to test the concepts in the exploratory factor analysis. It was an adequate sample size, scoring .811 in the Kaiser-MeyerOlkin Measure test. The EFA was performed using the maximum likelihood and promax rotation, with the number of components not defined in advance. The original model consisted of three concepts (constancy, confidence and control) with 14 items. However, after the EFA was conducted, it was clear that this would not be the case in this specific setting. The first suggested result was 4 factorial, with several items loading less than .4 (items 6, 9, 12). Item 1 did not load on any of the concepts. These items were therefore omitted, which resulted in 3-factorial solution and 10 items. The total variance reached $63.74 \%$ and there were no cross-loading or low loading items; the values reached between .426 and .868 for all items. However, two items switched place and loaded on different components than in the original model. Those items, 10 and 5, are discussed in more detail below.

Component 1 , originally called Confidence, consists of four items: 2, 3, 4, and 10. Item 10 was supposed to load on the component Constancy, but the nature of this research determined that it belongs in the Confidence component in this case. The wording of item 10 is as follows: "I do not get distracted easily and lose my 
concentration (reversed)". This component is concerned with the belief in being able to achieve goals and having superior skills to an opponent. Positive thinking is the key aspect of this component in the time of challenge and pressure (Sheard, 2010). This item was worded as "I have unshakeable confidence in my ability." Cronbach's alpha was tested and reached an acceptable result of .758 .

Constancy is the second component, measured by items 7, 8 and 5. Item 5, which reads "Under pressure, I am able to make decisions with confidence and commitment", originally loaded on the Confidence component. Concentration and responsibility are the main factors of this component; the willingness to be responsible for one's own goals and training and not give up despite complications. Furthermore, this component touches on the ability to remain focused even if one is provoked (Sheard, 2010). Cronbach's alpha here reached a questionable .687 .

The third component is called Control and does not differ from the original scale. It is composed of items 11, 13 and 14. Item 12 was omitted during the EFA, as stated above. The original wording of this component is "I do not get angry and frustrated when things do not go my way (reversed)". Those who score high on this component have good control over their emotions and are able to remain calm and relaxed when facing pressure. Also, when they find themselves in unexpected situations, they can rebuild their psychological control (Sheard, 2010). Cronbach's alpha reached .723, which is acceptable.

Table 1. Exploratory Factor Analysis of the Sport Mental Toughness Questionnaire

\begin{tabular}{|c|c|c|c|}
\hline \multirow[t]{2}{*}{ Items } & \multicolumn{3}{|c|}{ Factors } \\
\hline & Confidence & Constancy & Control \\
\hline 2. I have an unshakeable confidence in my ability & 0.849 & & \\
\hline 3. I have qualities that set me apart from other competitors & 0.674 & & \\
\hline 4. I have what it takes to perform well while under pressure & 0.611 & & \\
\hline 10. I get distracted easily and lose my concentration & 0.453 & & \\
\hline 5. Under pressure, I am able to make decisions with confidence and commitment & & 0.426 & \\
\hline 7. I am committed to completing the tasks I have to do & & 0.868 & \\
\hline 8. I take responsibility for setting myself challenging targets & & 0.635 & \\
\hline 11. I worry about performing poorly & & & 0.607 \\
\hline 13. I get anxious by events I did not expect or cannot control & & & 0.850 \\
\hline 14. I get angry and frustrated when things do not go my way & & & 0.674 \\
\hline Eigenvalue & 3.776 & 1.059 & 1.548 \\
\hline \begin{tabular}{|l|} 
Total variance \\
\end{tabular} & 37.661 & 10.598 & 15.485 \\
\hline Factor variance & 37.661 & 63.740 & 53.146 \\
\hline Reliability & 0.758 & 0.687 & 0.723 \\
\hline
\end{tabular}

\section{Confirmatory Factor Analysis}

On the second split of the sample $(n=139)$, the CFA was performed using AMOS software. The hypothesized model of three components and 10 items was tested with three different model fit indices. The parsimonious model fit was tested, complete with the incremental model fit indices. Incremental model fit indices compare the model on the continuum between the null model and the hypothesized model. Finally, absolute fit was tested, to see the difference between the model and the perfect fit.

The parsimonious model fit reached $\mathrm{x} 2 / \mathrm{df}=1.307$, with $\mathrm{x} 2=39.219$ and degrees of freedom equalling 30 . This result suggests a reasonably good fit 20 , when the 
$\mathrm{x} 2 / \mathrm{df}$ is less than .3. The incremental model fit showed a satisfactory fit; IFI $=965$, comparative fit index (CFI)

$=.963$ and $\mathrm{TLI}=.944$ suggest a satisfactory $\mathrm{fit}^{21}$. And finally, the root-mean-square error of approximation (RMSEA) reached .047, which is interpreted as a good fit (Browne \& Cudeck, 1993).

Table 2. Confirmatory Factor Analysis of Sport Mental Toughness Questionnaire.

\begin{tabular}{|c|c|c|c|c|c|c|c|c|c|}
\hline CMIN & DF & CMIN/DF & GFI & AGFI & NFI & TLI & IFI & CFI & RMSEA \\
\hline 39.219 & 30 & 1.307 & .947 & .902 & .866 & .944 & .965 & .963 & .047 \\
\hline
\end{tabular}

CMIN: $x^{2}$; DF: de gree of freedom; GFI: goodness-of-fit index; AGFI: adjusted GFI; NFI: normed fit index; TLI: Tucker-Lewis index; IFI: incremental fit index; CFI: comparative fit index; RMSEA: root mean square error of approximation.

While the original model of mental toughness created by Sheard et al. (2009) and his team served as a valid base for this research, adjustments are required in order to be applicable to modern and traditional wushu. After the deletion of four items and switching two items on two components, the model fit is good and therefore better suitable for studies of wushu.

\section{Discussion}

The purpose of the study was to evaluate the Sport Mental Toughness in the setting of Chinese martial arts, specifically targeted to adult European practitioners and athletes. An EFA was conducted and resulted in the omission of four items due to low loading, and two items switched their places in relation to the components. The internal consistency of the components reached acceptable results, however the Constancy component yielded questionable values (.687). The new model was tested with CFA and the model fit was confirmed as a good fit.

The two items which were switched from their original components are items 10 and 5. Item 10, "I do not get distracted easily and lose my concentration (reversed)" originally belonged to the Constancy component, meaning the individual is able to retain his or her focus for a long period of time; the emphasis is on constant focus. This research has determined that this item instead loads on the Confidence component. This component is connected with the ability to achieve goals, maintain positive thinking and being confident about having superior skills to rivals. This suggest that the first part of the item, being easily distracted (with emphasis on the word easily) may be perceived by the respondents as questioning their belief in their abilities of keeping focused, not on the focus itself.
A long career is typical for martial arts practitioners. This long-term prospect of one's training may increase the necessity of positive thinking. Moreover, there is no standardized belt ranking in Chinese martial arts, unlike in karate, for example. The practitioner continues training with the only visible milestone when he or she starts teaching or assisting in teaching. There are no levels that would satisfy one's need for accomplishment, which may be quite a difficult situation to handle in the longterm. That may be paired with the following component, Constancy.

Item 5, "Under pressure, I am able to make decisions with confidence and commitment" was originally part of the Confidence component, but now has been placed within the Constancy component. The wording perhaps resonated among the respondents with their ability to keep their focus constant, even under pressure. Thus, they might have understood this question as the ability to maintain their decision-making skills in any circumstance.

Croom (2014) emphasized in his work the skills one gains through partner exercises, such as sparring, where body-to-body contact is utilized. The presence of physical intimacy and often pain of the practitioner and the partner can be interpreted as a kind of pressure to be dealt with repeatedly. Thus, martial arts practitioners need to keep focused even in this case, since losing concentration can result in hurting the partner or self.

Control, the third component that remains unchanged, is described as the ability to rebuild the psychological control when under pressure and when a situation changes unexpectedly, as well as controlling emotions. The psychological control is an important skill in Chinese martial arts: Bowman (2016) described the process of 
learning techniques of Japanese and Korean martial arts, and how the student slowly starts to understand the principles of the biomechanics; in terms they are similar. But in the case of Chinese martial arts, it is "becoming animal. You are tiger. Then you are eagle." (p. 88). The practitioners use their imagination and visualisation to guide and improve their movements, which corresponds with the complex movements of an animal, and thus there is great demand on their psychological control. Moreover, while fighting, the situation is changing constantly, and practitioners have to adapt to this while remaining calm and relaxed, so they are able to execute their defence or attack quickly and precisely.

This model of three components was supported, which is a significant addition to the discussion of unidimensionality of mental toughness. Gucciardi, et al. (2015) introduced the model consisting of only one dimension, due to the overlap of the components. This was a milestone in mental toughness research, since previous works were always based on multiple components. However, this paper does not support the unidimensionality, as it supports three components of clearly separate loadings. Nevertheless, the internal consistency of Constancy requires further attention, preferably utilizing a larger sample size and perhaps involving participants outside of Europe. For example, in America there is an extended population of both modern and traditional wushu practitioners, and therefore a similar study there with the comparison of these results would be beneficial for the field, and would have benefits beyond the internal consistency of the one specific component.

\section{Conclusions}

This paper tested the mental toughness model in the Chinese martial arts setting. Mental toughness has previously been explored in various sports, however, there is a lack of research focusing on martial arts in general and Chinese martial arts specifically. This paper therefore contributes to the narrowing of this gap. Martial arts practitioners face challenges that other sports practitioners may not face, and therefore the model of mental toughness needs to be tailored to these populations directly.
Moreover, this paper significantly contributes to the discussion on the multidimensionality of mental toughness, as opposed to the recently proposed unidimensional model. This study supports the evidence for multidimensionality of mental toughness with three components.

The limitation of this research is surely its sample size. The number of practitioners of traditional wushu is extensive, but the number of adult modern wushu practitioners is less so. For that reason, only 111 modern wushu practitioners were sampled. It is nevertheless believed that this is a great portion of the existing modern wushu practitioner population in Europe.

The findings of this research can be applied to the development of mental skills training program for martial arts in general. With the demands placed on practitioners, in terms of performance, fighting, pain and body contact, this is certainly an important area to develop. The model developed in this study may be used for further research, leading to improved mental skills training in martial arts programs. Finally, it is still necessary to test the model in other geographic and cultural environments, and the comparison to this study may bring further research directions.

\section{References}

Awang, Z. (2012). Structural equation modeling using AMOS graphic. Penerbit Universiti Teknologi MARA.

Bowman, J. (2016). Mythologies of martial arts. Rowman $\&$ Littlefield International.

Browne, M. W., \& Cudeck, R. (1993). Alternative ways of assessing model fit. Sage focus editions, 154, $136-136$

Bull, S. J., Shambrook, C. J., James, W., \& Brooks, J. E. (2005). Towards an understanding of mental toughness in elite English cricketers. Journal of applied sport psychology, 17(3), 209-227.

Chen, M. A., \& Cheesman, D. J. (2013). Mental toughness of mixed martial arts athletes at different levels of competition. Perceptual and motor skills, $116(3), 905-917$. 
Clough, P., Earle, K., \& Sewell, D. (2002). Mental toughness: The concept and its measurement. Solutions in sport psychology, 32-43.

Croom, A. M. (2014). Embodying martial arts for mental health: Cultivating psychological wellbeing with martial arts practice.

Gucciardi, D. F., Hanton, S., Gordon, S., Mallett, C. J., \& Temby, P. (2015). The concept of mental toughness: tests of dimensionality, nomological network, and traitness. Journal of Personality, 83(1), 26-44.

Heiny, K. A. (2012). Psychometric analysis and dimensionality of mental toughness in the context of judo performance (Doctoral dissertation, uniwien).

International Wushu Federation. (n.d.). Retrieved May 18. 2018, from http://www.iwuf.org/wushu/2014/0926/183. $\mathrm{html}$

Jones, G. (2002). What is this thing called mental toughness? An investigation of elite sport performers. Journal of applied sport psychology, 14(3), 205-218.

Kline, R. B. (1998). Software review: Software programs for structural equation modeling: Amos, EQS, and LISREL. Journal of psychoeducational assessment, $16(4), 343-364$

Kobasa, S. C. (1979). Stressful life events, personality, and health: an inquiry into hardiness. Journal of personality and social psychology, 37(1), 1 .

Kuan, G., \& Roy, J. (2007). Goal profiles, mental toughness and its influence on performance outcomes among Wushu athletes. Journal of sports science \& medicine, 6(CSSI-2), 28.

Loehr, J. (1982). Mental toughness training for sports: Achieving athletic excellence. Plume.

Lorge, P. A. (2012). Chinese martial arts: From antiquity to the twenty-first century. Cambridge University Press.
Middleton, S. C., Marsh, H. W., Martin, A. J., Richards, G. E., \& Perry, C. (2004). Discovering mental toughness: A qualitative study of mental toughness in elite athletes [R].

Minnix, D. W. (2010). Mental Toughness in the Classical Martial Arts (Doctoral dissertation, Virginia Tech).

Schafer, J. L. (1999). Multiple imputation: a primer. Statistical methods in medical research, 8(1), 3-15.

Sheard, M. (2012). Mental toughness: The mindset behind sporting achievement. Routledge.

Sheard, M., Golby, J., \& Van Wersch, A. (2009). Progress toward construct validation of the Sports Mental Toughness Questionnaire (SMTQ). European Journal of Psychological Assessment, 25(3), 186-193.

Singh, K., M. (2015). Analysis of Mental Toughness and Mental Health of Tae-Kwon-Do and Judo Players. IJAR, 1(9), 507-510.

Thelwell, R., Weston, N., \& Greenlees, I. (2005). Defining and understanding mental toughness within soccer. Journal of Applied Sport Psychology, 17(4), 326-332.

Wang G. (2010). Chinese Kungfu. Masters, schools and combats. China intercontinental press.

Note :

Conflicts of interest. The author certify that there is no conflict of interest with any financial organization regarding the material discussed in the manuscript.

Funding. This project did not receieve any funding, and was conducted as part of a $\mathrm{PhD}$ project at Hong Kong Baptist University.

\section{Correspondence}

Veronika Partikova,

Department of Sport and Physical Education,

Hong Kong Baptist University, Hong Kong.

E-mail:vpartikova@gmail.com 